\title{
Feedforward augmented Sliding Mode Motion Control of Antagonistic Soft Pneumatic Actuators
}

\author{
Erik H. Skorina, Ming Luo, Selim Ozel, Fuchen Chen, Weijia Tao, and Cagdas D. Onal
}

\begin{abstract}
Soft pneumatic actuators provide many exciting properties, but controlling them without the use of bulky and expensive flow-control valves can be difficult and computationally expensive. We seek a solution to this problem by introducing an inexpensive and reliable muscle-like linear soft actuator used antagonistically to operate a rigid 1-DoF joint, resulting in a system that combines the advantages of rigid and soft robotics. Using this setup, we performed precise motion control using a sliding mode feedback controller as well as a sliding mode controller augmented by a feedforward term to modulate the state of solenoid valves that drive each actuator. We found that both controllers performed equivalently well in following a step function and in responding to a disturbance. The feedforward augmented controller performed significantly better when following dynamic trajectories over a range of frequencies and with the addition of an external force. The next step will be to modify our valve control scheme to allow for the determination of both the position and stiffness of the joint, better leveraging the advantages of soft pneumatic actuators.
\end{abstract}

\section{INTRODUCTION}

Soft pneumatic actuators first emerged in the 1950s with the development of the McKibben Artificial Muscle [1], [2], [3], [4], [5]. Fluidic Elastomeric Actuators [6], [7], [8], [9], [10] were developed recently to advance the applications of soft pneumatic actuators towards a bio-inspired soft robotic vision. While soft actuators provide many exciting properties, these properties are largely academic without the ability to control them with precision and repeatability. The control of soft pneumatic actuators, which would enable more practical applications for wider adoption, is a topic that is only recently being addressed.

Motion control of pneumatic soft actuators remains a challenge. The dynamic behavior of these actuators includes a nonlinear and non-trivial time delay as pressurized air is introduced through valve commands. Moreover, since flowcontrol valves are bulky and expensive, the solenoid valves commonly available offer only two operational states: on and off. This makes it difficult to precisely control the pressure or flow rate of air inside each actuator.

In this paper, we introduce the advanced motion control of a simple 1 degree of freedom (DoF) revolute joint driven by a new class of pneumatic soft actuator that combines the properties of the McKibben Actuator and the Fluidic Elastomeric Actuators. Figure 1 displays the proposed pneumatic soft linear actuator. Similar to McKibben actuators, these elastomeric actuators have two symmetrical helical fibers,

The authors are with the Mechanical Engineering Department and Robotics Engineering Program, Worcester Polytechnic Institute, MA 01609, USA. All correspondence should be addressed to Cagdas D. Onal cdonal@wpi.edu

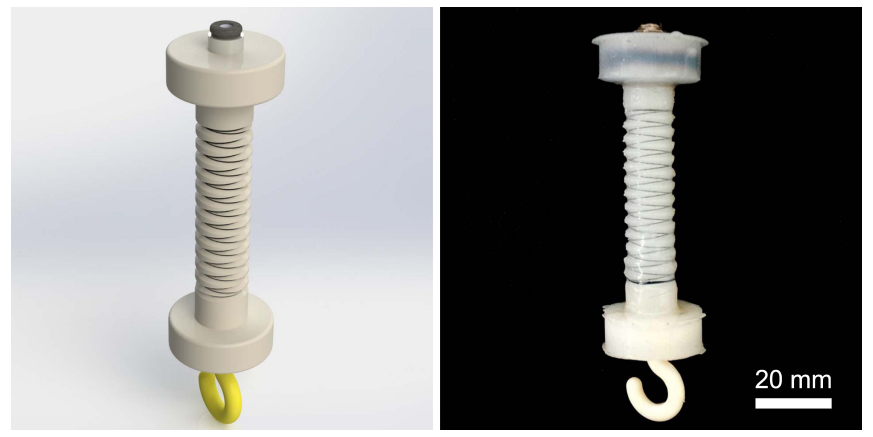

Fig. 1. A CAD model (left) and a physical prototype (right) of the proposed soft pneumatic linear actuator, which offers convenient physical and fluidic connectors to operate rigid kinematic linkages.

which create geometrical constraints under pressure to ensure that the actuator only undergoes axial deflection. They are inexpensive, while allowing for straightforward and rapid fabrication. In addition, the case study detailed in this paper provides a realistic application of soft actuators to operate rigid kinematic systems in an antagonistic arrangement (as seen in Figure 3). Our approach is to operate a 1-DoF revolute joint, as a representative system, controlled precisely using only one solenoid valve for each soft actuator.

While motion control of the recently developed soft pneumatic actuators has little precedence, literature describes specialized methods of controlling traditional rigid pneumatic piston actuators. Due to valving hardware limitations, the general approach is to utilize the duty cycle of a pulse width modulation (PWM) signal of solenoid valves to regulate position errors. Gentile et al. [11] proposed a proportional integrative (PI) controller with position feedforward for linear position control. Varseveld et al. [12] proposed a proportional integral derivative (PID) controller with added friction compensation and position feed-forward for the piston position control. Shih et al. [13] applied fuzzy control and a modified differential PWM to control the position of a pneumatic rodless cylinder. However, this method needs to determine a membership function and fuzzy rules, which increases complexity and reduces repeatability. Kyoungkwan et al. [14] proposed a switching algorithm for control parameters using a learning vector quantization neural network. In addition, [15], [16], [17] control the piston using an analytical model. This model is based on the whole piston system, and not a single actuator. A sliding mode control approach was presented in [16] and [17], which requires multiple solenoid valves for each actuator, 


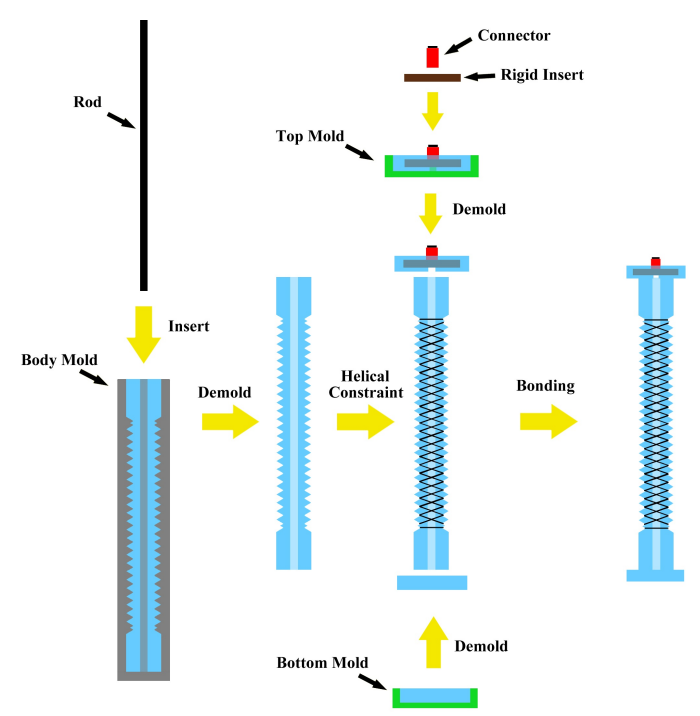

Fig. 2. The fabrication process of the proposed soft linear actuator.

increasing complexity.

Based on our prior work in this space, we present two different controllers to address the motion control problem of soft actuation. The first one is a sliding mode controller [18], while the second one combines the developed sliding mode controller with a static mapping function to create a feedforward augmented sliding mode $(\mathrm{SM}+\mathrm{FF})$ controller. Experimental results indicate that both controllers are capable of achieving high levels of precision, but that the SM+FF controller is more effective, especially for dynamic trajectory following scenarios and under a payload.

The contributions of this work include:

- Introduction of an inexpensive and reliable muscle-like linear soft actuator that can be driven by larger pressure values compared to earlier prototypes;

- Development of a benchtop setup to test soft actuator control on rigid kinematic mechanisms, resulting in a realistic system combining the advantages of rigid and soft robotic approaches; and

- Precise motion control of a soft actuation system using two different control techniques by the modulation of one solenoid valve for each actuator.

This paper starts with a description the design and fabrication of the soft actuator. It continues with a discussion of the properties of the 1-DoF joint we used to test the controllers. Next, it describes the problem formulation, including a lumped dynamic model of the soft linear actuator and the derivation of the two controllers. Finally we will describe our experimental findings and conclude the paper.

\section{DESIGN AND FABRicATION}

\section{A. Fabrication of the soft actuator}

The presented soft linear actuator was made by molding silicone rubber (Smooth-on Dragonskin 10) in a 3-D printed mold. The step-by-step fabrication process (shown in Fig. 2) is described below:

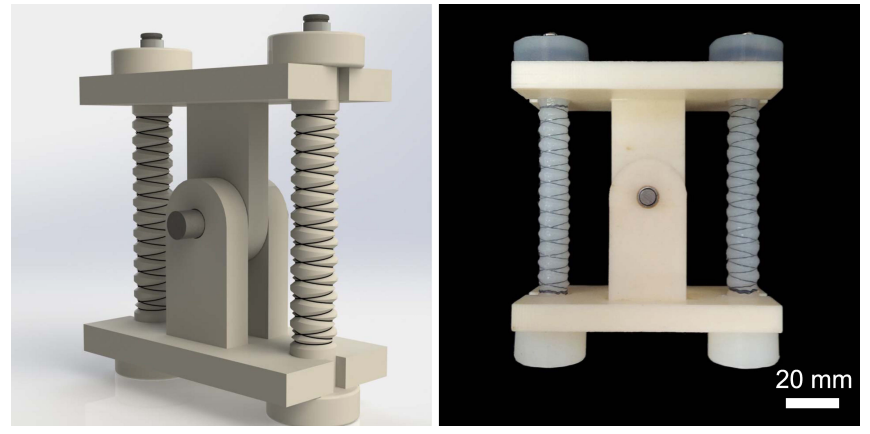

Fig. 3. A CAD model (left) and a physical prototype of the 1-DoF revolute joint operated by the proposed soft actuators in antagonism.

Step 1: Insert a carbon fiber or metallic rod of appropriate diameter into the center of the body mold to create the hollow cylindrical core inside the actuator. Introduce silicone rubber into the body mold with the rod inside, the top connector mold and the bottom mold.

Step 2: After silicone rubber has cured, remove the rod inside the body mold, then remove the silicone from the body mold.

Step 3: Tie two symmetrical helices of thread around the cylindrical silicone rubber body guided by the grooves. Apply an outer layer of uncured silicone rubber around the threads to permanently bond the thread to the actuator.

Step 4: Embed the connector into the top mold just before the silicone rubber had fully cured. Once cured, remove the top and bottom silicone rubber pieces from their respective molds. Bond these to the silicone rubber body with a thin layer of uncured silicone rubber.

\section{B. Joint Design}

As a benchtop test setup, we developed a simple 1-DoF revolute joint. As the proposed soft linear actuators only work in extension, bidirectional operation necessitates the use of two actuators in antagonism. The final prototype can be seen in Figure 3. In addition, an optical encoder (CUI Inc. AMT 203) was mounted on the rotation axis to offer reliable joint angle feedback for closed loop control.

The joint is designed to be longer than the nominal length of the actuators, forcing them to be pre-strained during assembly. This prevents the actuators getting in the way of joint movement when not under pressure. Without a prestrain that keeps the soft actuators under tension, the joint will collide with an actuator during operation, forcing it to compress and buckle outwards, and reducing the mobility of the joint. The original threaded length of the actuators are approximately $50 \mathrm{~mm}$, which is stretched to be around 75 $\mathrm{mm}$, for a pre-strain of $50 \%$.

\section{Antagonistic Solenoid Valve Operation}

Each of the two actuators controlling the joint were connected to a 20 psi [138 $\mathrm{kPa}$ ] pressurized air line and 


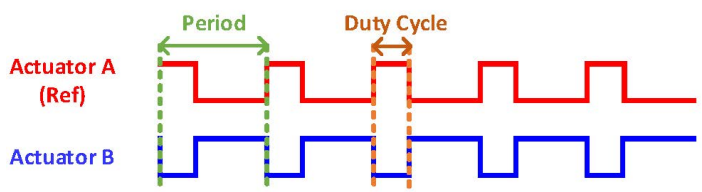

Fig. 4. The two PWM control signals of the two actuators are opposite.

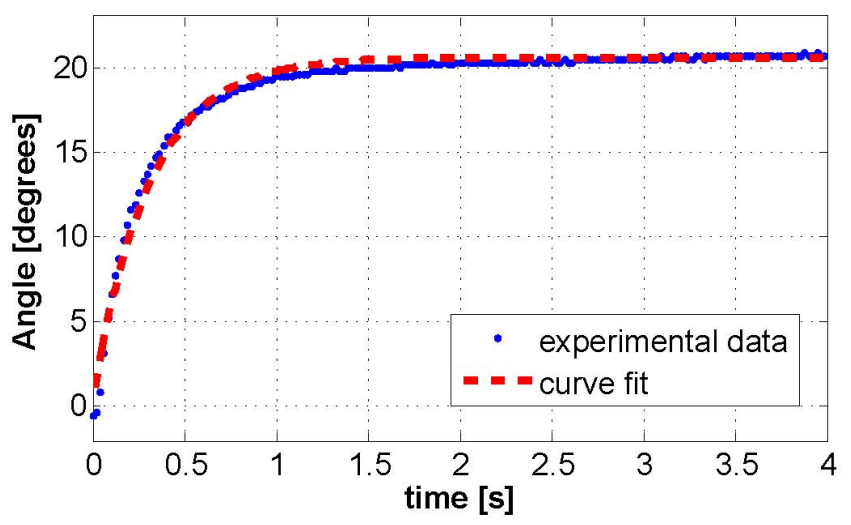

Fig. 5. The experimental data from a representative duty cycle (65\%) step input and its corresponding second order dynamic curve fit.

each controlled by a separate solenoid valve. To approximate pressure inputs between 0 and $20 \mathrm{psi}[138 \mathrm{kPa}$ ], we operated the valves with a $30 \mathrm{~Hz}$ PWM signal. This resulted in a 1-DoF system being controlled by two separate inputs. To simplify joint control, we created a antagonistic scheme, where the valves are always in opposition to each other as depicted schematically in Fig. 4. Thus, if Valve A has a PWM signal with a duty cycle of $70 \%$, Valve B will have a duty cycle of $30 \%$. To control the joint angle, we specify a single number between 0 and 100 corresponding to the duty cycle of Valve A.

\section{PRoblem Formulation}

\section{A. Sliding Mode Controller Design based on Lumped System Dynamics}

Relying on electrical circuit equivalence of pneumatic systems [9], the dynamic behavior of the 1-DoF joint can be approximated as a lumped second order dynamic equation as:

$$
\theta=C_{1} e^{(-t / \tau 1)}+C_{2} e^{(-t / \tau 2)}+C_{0}
$$

Where $C_{1}, C_{2}$ are constant coefficients, $\tau_{1}, \tau_{2}$ are time constants, and $C_{0}$ is the steady-state static angle, with a one-toone relation to the PWM duty cycle, indicating the angle the joint will converge after the dynamic terms dissipate. Figure 5 displays results of fitting equation (1) on experimental step pressure input data at $65 \%$ duty cycle.

The dynamic response of the actuator (1) can then be described as the second order system:

$$
\widehat{a} \ddot{\theta}+\widehat{b} \dot{\theta}+\theta=\widehat{c} u(t)
$$

where $\theta$ is the rotation angle with respect to time, $u(t)$ is the input Duty Cycle of the PWM signal and $\widehat{a} \in$
TABLE I

Coefficients of Soft Actuator Dynamic Model under Varying DUTY CYCLES (95\% CONFIDENCE INTERVALS)

\begin{tabular}{llllll}
\hline \hline$a_{o}$ & $b_{o}$ & $c_{o}$ & $\Delta a$ & $\Delta b$ & $\Delta c$ \\
0.2332 & 1.3588 & 1.4474 & 0.2476 & 1.0165 & 0.5170 \\
\hline \hline
\end{tabular}

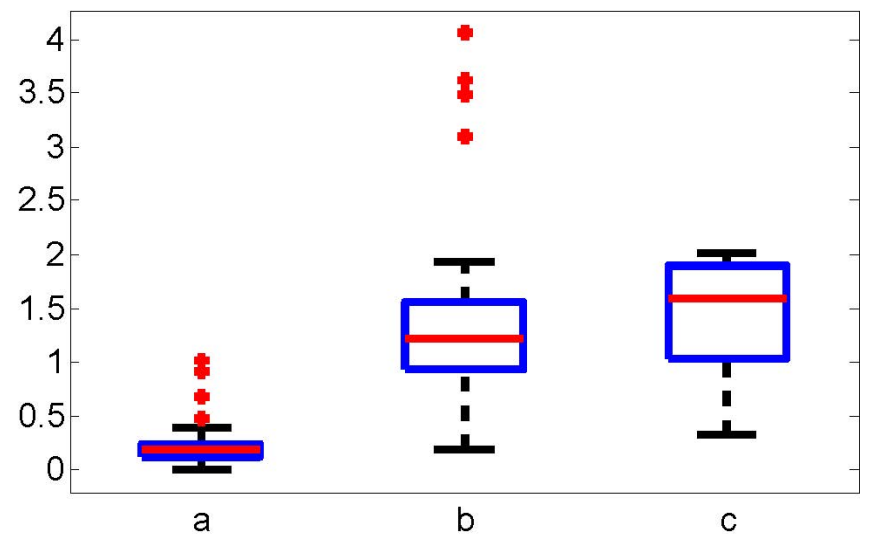

Fig. 6. A box plot representation of the dynamic coefficients for joint response to duty cycles of $35 \%$ to $65 \%$ under $20 \mathrm{psi}[138 \mathrm{kPa}$. The red line is the mean, the blue box is 1 standard deviation, the black lines are the $95 \%$ confidence bounds, and the red crosses are outliers.

$\left(a_{o}-\Delta a, a_{o}+\Delta a\right), \widehat{b} \in\left(b_{o}-\Delta b, b_{o}+\Delta b\right)$ and $\widehat{c} \in$ $\left(c_{o}-\Delta c, c_{o}+\Delta c\right)$ are bounded uncertainty parameters. $a_{o}, b_{o}$ and $c_{o}$ and $\Delta a, \Delta b$ and $\Delta c$ are the mean and $95 \%$ confidence interval values of $a, b, c$. The Figure 6 shows the variation of parameters $\widehat{a}, \widehat{b}$ and $\widehat{c}$ for duty cycles ranging from $35 \%$ to $65 \%$ under 20 psi [138 $\mathrm{kPa}$ ] pressure input. Table I shows the extracted values of these parameters.

Equation (2) can be rewritten in state-space form as:

$$
\dot{\boldsymbol{x}}=\boldsymbol{A} \boldsymbol{x}+\boldsymbol{B} u(t)
$$

where $\boldsymbol{x}=[\theta, \dot{\theta}]^{T}, \boldsymbol{A}=\left[\begin{array}{cc}0 & 1 \\ -\frac{\widehat{b}}{\widehat{a}} & -\frac{1}{\widehat{a}}\end{array}\right] \boldsymbol{B}=\left[\frac{\widehat{c}}{\widehat{a}}, 0\right]$. Thus, an iterative sliding mode controller can be designed for motion control based on the given dynamic model and bounded uncertainty parameters. For a given reference $x^{r e f}$, the position error is given as:

$$
e_{x}=\boldsymbol{G}_{\boldsymbol{x}}\left(\boldsymbol{x}^{r e f}-\boldsymbol{x}\right),
$$

where $\boldsymbol{G}_{\boldsymbol{x}}=[C, 0]$. We define a non-negative Lyapunov function candidate and its derivative as follows:

$$
\begin{gathered}
V_{x}=\frac{e_{x}^{2}}{2} \geq 0 \\
\dot{V}_{x}=e_{x} \dot{e_{x}},
\end{gathered}
$$

and select a desired dynamic error manifold as:

$$
\dot{e_{x}}+D_{x} e_{x}=0
$$

where $\boldsymbol{D}_{\boldsymbol{x}}$ is a positive constant. Plugging (7) into (6), we obtain a non-positive Lyapunov function derivative as:

$$
\dot{V}_{x}=-D_{x} e_{x}^{2} \leq 0,
$$




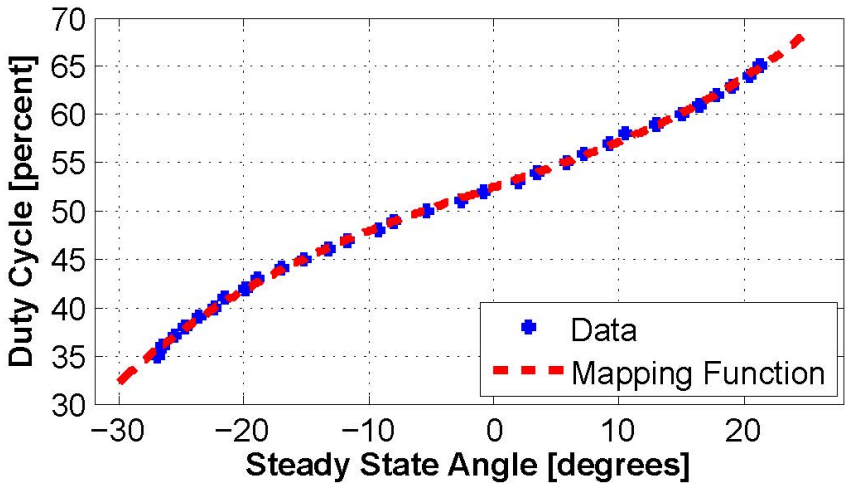

Fig. 7. Steady state angle response at various duty cycles and the corresponding mapping mapping function. Adjusted $R^{2}$ value for the fit is 0.9993 .

which will ensure stability. Combining (3) and (4),

$$
\begin{array}{rl}
\dot{e_{x}} & =\boldsymbol{G}_{\boldsymbol{x}}\left(\boldsymbol{x}^{\dot{\boldsymbol{r} e f}}-\dot{\boldsymbol{x}}\right) \\
& =\boldsymbol{G}_{\boldsymbol{x}}\left(\boldsymbol{x}^{\dot{\boldsymbol{r}} \boldsymbol{f}}-\boldsymbol{A} \boldsymbol{x}-\boldsymbol{B} u(t)\right) \\
& =\boldsymbol{G}_{\boldsymbol{x}} \boldsymbol{B}\left(u_{e q}(t)-u(t)\right), \\
\boldsymbol{G}_{\boldsymbol{x}} & \boldsymbol{B}\left(u_{e q}(t)-u(t)\right)+D_{x} e_{x}=0,
\end{array}
$$

where $u_{e q}(t)$ is the continuous equivalent control input, which is difficult to calculate [19]. Rearranging (9) reveals:

$$
u_{e q}(t)=u(t)+\left(\boldsymbol{G}_{\boldsymbol{x}} \boldsymbol{B}\right)^{-\mathbf{1}} \dot{e_{x}} .
$$

Approximating $u_{e q}(t)$ with $u_{e q}(t-\Delta t)$, where $\Delta t$ is the time step yields the iterative sliding mode control law:

$$
u(t)=u(t-\Delta t)+\left(\boldsymbol{G}_{\boldsymbol{x}} \boldsymbol{B}\right)^{-\mathbf{1}}\left(\dot{e_{x}}+D_{x} \boldsymbol{e}_{\boldsymbol{x}}\right)
$$

For simplicity, we define $K=\left(\boldsymbol{G}_{\boldsymbol{x}} \boldsymbol{B}\right)^{-\mathbf{1}}$ as a scalar positive tuning factor as:

$$
u(t)=u(t-\Delta t)+K\left(\dot{e_{x}}+D_{x} e_{x}\right) .
$$

\section{B. Feed Forward Controller Design}

In order to improve this control algorithm, we made use of the fact that a given control input converges to a single steady state angle. We experimentally determined these input-output combinations and fit a 3rd order polynomial to the data that returned the approximate antagonistic PWM duty cycle to reach a given angle, as shown in Figure 7 . We then incorporated this mapping function to add a feedforward term to the sliding mode controller $(\mathrm{SM}+\mathrm{FF})$ as:

$$
u_{h}(t)=\operatorname{Map}\left(\theta^{\text {ref }}\right)+u(t)
$$

In this equation, $u_{h}(t)$ is the $\mathrm{SM}+\mathrm{FF}$ output signal, $u(t)$ is the controller signal from (13), and $\operatorname{Map}\left(\theta^{\text {ref }}\right)$ is the mapped PWM duty cycle, which results in the reference angle in steady state. Substituting in, we obtain:

$$
u_{h}(t)=u(t-\Delta t)+K\left(\dot{\boldsymbol{e}_{\boldsymbol{x}}}+D_{x} \boldsymbol{e}_{\boldsymbol{x}}\right)+\operatorname{Map}\left(\theta^{\text {ref }}\right)
$$

\section{EXPERIMENTAL RESULTS}

We first tested our controllers against a step reference, displayed in Figures 8 and $9^{1}$. The control loop was run at

\footnotetext{
${ }^{1}$ These experiments and others can be seen in our attached video.
}

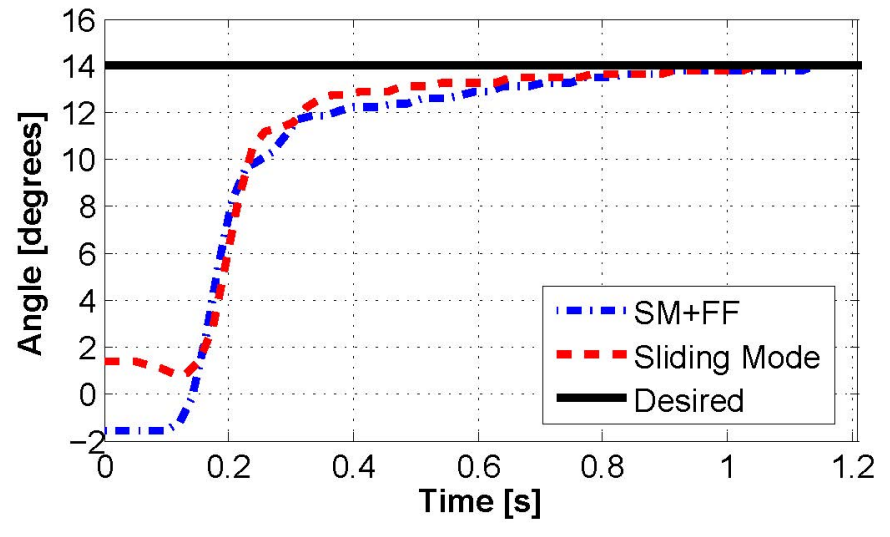

Fig. 8. A comparison of the two control algorithms on a step response. Small variations in the starting point of each trial are a result of frictional effects.
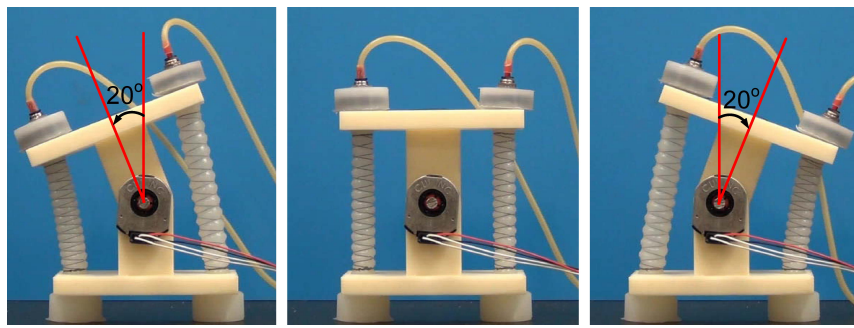

Fig. 9. The feedforward assisted sliding mode controller offers precise position control under step reference signals in both directions. As they are pre-strained, the actuators are never under compression.

$38 \mathrm{~Hz}$, which was sufficiently fast when compared to our system response time. As shown in Fig. 8, the differences between the Sliding Mode and SM+FF controller for this simple case are trivial. The control coefficients used for all experimental results were tuned through preliminary experiments to be $K=30$ and $D_{x}=0.0033$.

Next, we tested our controllers with a more complicated signals: offset sinusoids with frequencies between 0.2 and $1 \mathrm{~Hz}$.Experimental results of these trials can be seen in Figures 10 for the 0.2 and $1 \mathrm{~Hz}$ sinusoidal waves. These figures indicate that the sliding mode controller follows a sinusoidal trajectory with a constant time delay with respect to the reference signal. At lower frequencies, it follows the input signal with a slight lag, while at higher frequencies it lags behind with a reduced amplitude due to the slow dynamic characteristics of the soft actuators.

The SM+FF controller, on the other hand, follows a more unique path. At lower frequencies, it catches up with the input signal before each peak and overshoots slightly. Though its amplitude is higher than the reference signal, the $\mathrm{SM}+\mathrm{FF}$ response is minimally phase shifted at lower frequencies, providing a level of compensation for slow actuator dynamics. At higher frequencies, the SM+FF controller can no longer catch up with the reference signal, only slightly less phase shifted than the sliding mode controller, but it offers a more accurate amplitude.

Figure 12 shows a plot the root-mean-square (RMS) error 

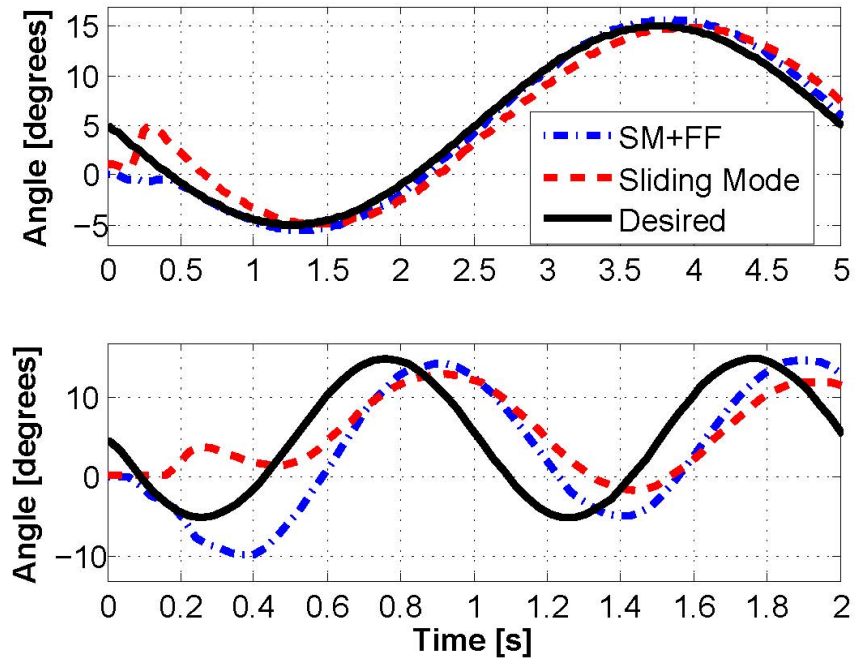

Fig. 10. A comparison of the two control algorithms tracking $0.2 \mathrm{~Hz}$ (top) and $1 \mathrm{~Hz}$ (bottom) sinusoidal waves.
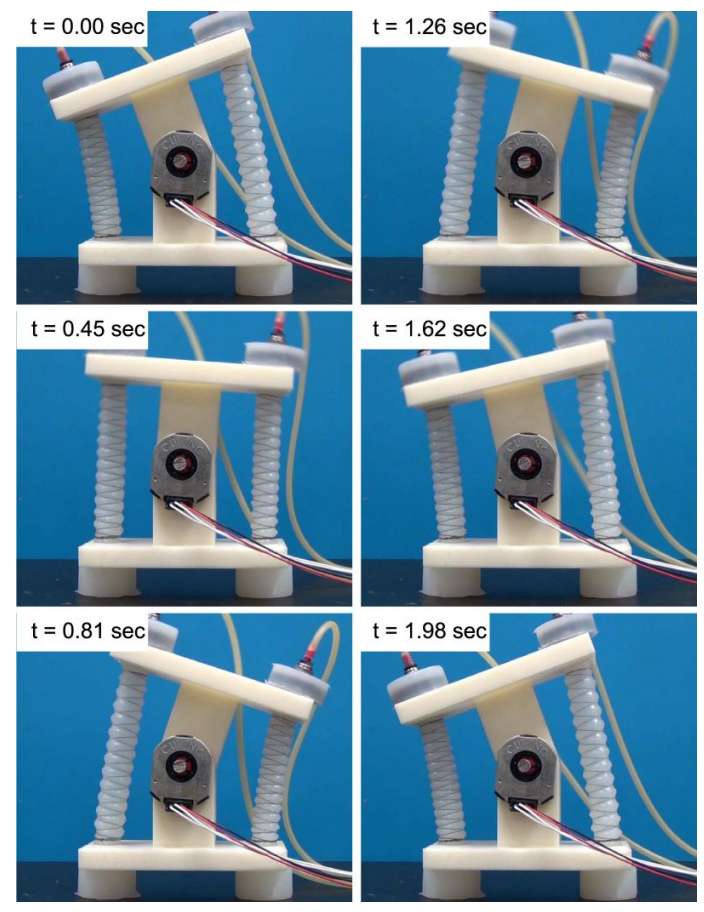

Fig. 11. A series of snapshots taken from a $0.5 \mathrm{~Hz}$ sinusoidal wave tracking experiment using the $\mathrm{SM}+\mathrm{FF}$ controller.

and phase shift of the two controllers over the given range of sinusoidal frequencies. Both values increase with frequency, but the SM+FF controller maintains lower tracking errors and phase differences for all frequencies tested.

In addition, to test the robustness of our controllers, we performed the same low-frequency experiment with a $200 \mathrm{~g}$ weight pulling perpendicular to the joint in the positive angular direction to quantify the performance of the $\mathrm{SM}+\mathrm{FF}$ controller when the mapping function is disturbed through external loading (see Figure 13). The results of this experiment can be seen in Figure 14, which indicates that,
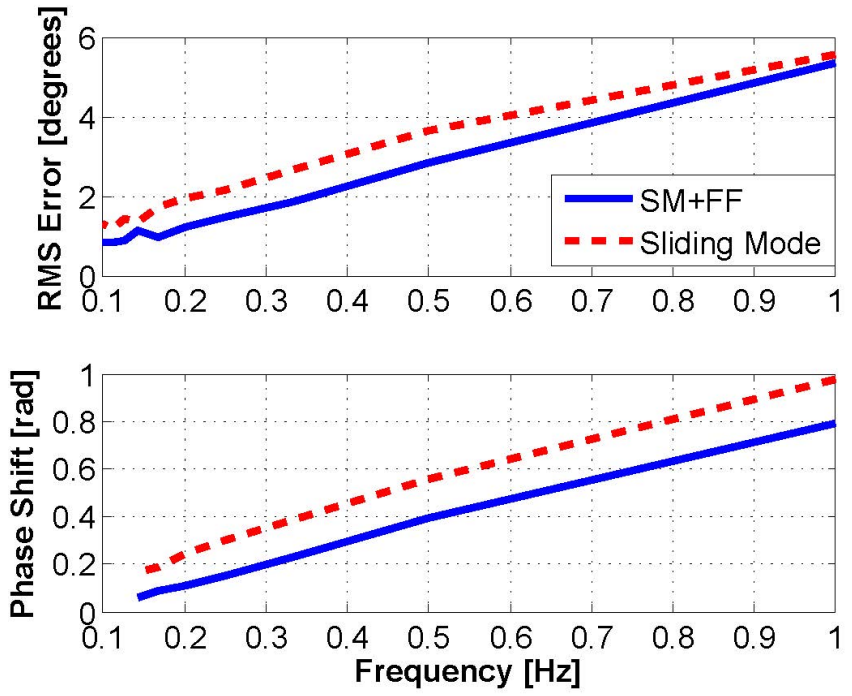

Fig. 12. Root mean square (RMS) tracking error (top) and the corresponding phase shift values (bottom) of tracking sinusoidal waveforms of a range of frequencies.
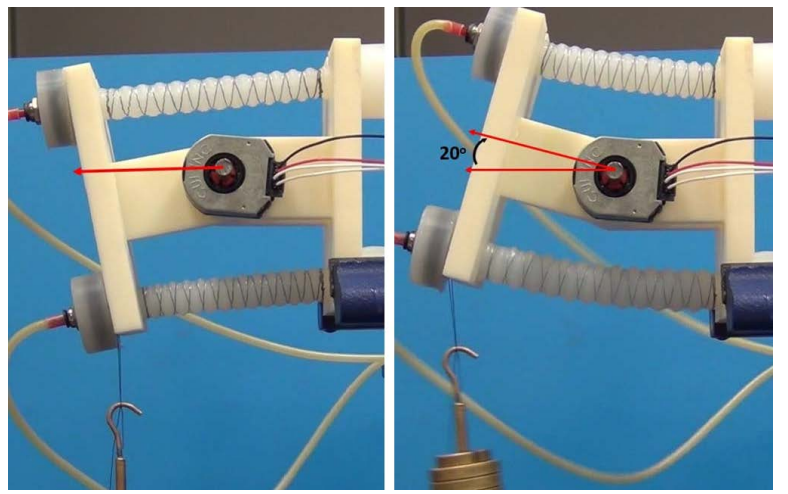

Fig. 13. Step reference tracking using the SM+FF controller under a $200 \mathrm{~g}$ payload.

even with an incorrect mapping the $\mathrm{SM}+\mathrm{FF}$ controller still outperforms the sliding mode feedback controller.

Finally, we explored the ability of the controllers to respond to a sudden disturbance, performed similarly to the previous experiment. For these experiments, we utilized a step reference signal and added the $200 \mathrm{~g}$ payload shortly after the set point is reached. The results of this test can be seen in Figure 15, which demonstrates that the two controllers respond similarly, with limited improvement from the $\mathrm{SM}+\mathrm{FF}$ controller.

\section{COnClusion And Future Work}

In this paper we discussed the design and fabrication of a new generation of soft pneumatic linear actuators and developed a 1-DoF revolute joint driven by two of these actuators antagonistically. We also derived a sliding mode feedback controller for controlling a this joint, then combined it with a static mapping function to provide feedforward assistance to the sliding mode controller $(\mathrm{SM}+\mathrm{FF})$. The $\mathrm{SM}+\mathrm{FF}$ controller was notably more effective in following 


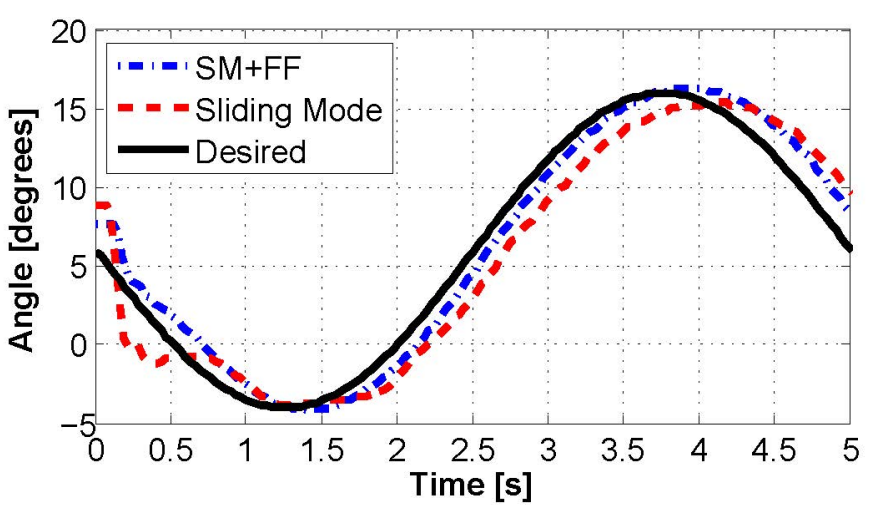

Fig. 14. A comparison of the two control algorithms following a sine wave with an external torque of $0.1 \mathrm{~N}-\mathrm{m}$ acting in the positive direction.

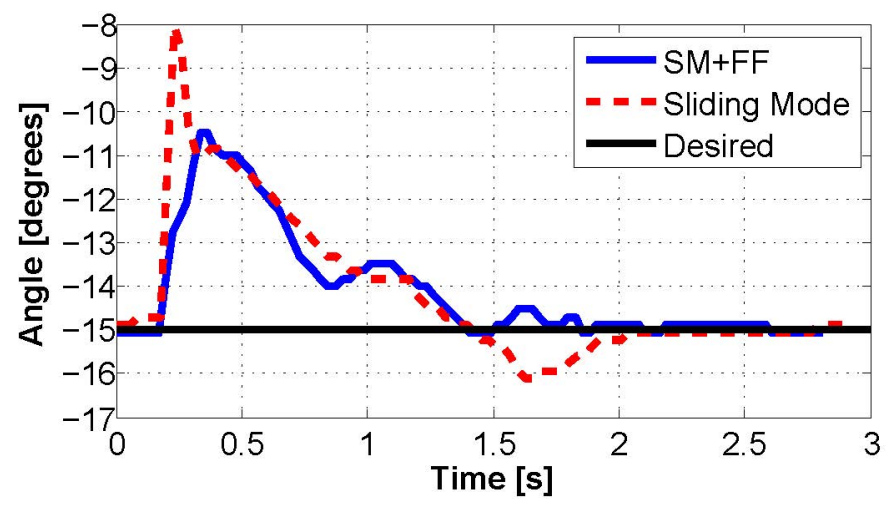

Fig. 15. Experimental comparison of the two control algorithms when a $200 \mathrm{~g}$ weight is added as a sudden disturbance after the step function has been reached.

a continuous trajectory even under external loading and disturbance, while the SM+FF and sliding mode controllers performed similarly in following a step function without payload.

The SM+FF controller is easy to implement, and requires little additional computational complexity, based on the characterization of the static response of the soft actuator driven joint under a range of pressure inputs supplied by varying PWM duty cycles. For this small cost, it gains significant accuracy in dynamic signal tracking. The only shortcomings of this approach are the requirement of the initial calibration step for each new actuator (which is simple and can be automated), and that potential shifts in the actuator attachment point may change the static relation slightly, though we demonstrated it still showed improvements. The need to recalculate the mapping function may be circumvented by a higher-fidelity model of actuator behavior, allowing the mapping to be calculated theoretically.

Additionally, future work includes further refining the proposed $\mathrm{SM}+\mathrm{FF}$ controller, combining the calibrated system dynamics with the static mapping function for a more reliable dynamic feedforward term. Finally, our future plans include the development of a 2-DoF shoulder joint driven by multiple soft linear actuators. We can modify our controller to enable for full 2-DoF control. Arrangements of these rigid kinematic systems will be used as part of a robotic arm fully driven by soft linear actuators with controllable stiffness.

\section{REFERENCES}

[1] C.-P. Chou and B. Hannaford, "Measurement and modeling of mckibben pneumatic artificial muscles," Robotics and Automation, IEEE Transactions on, vol. 12, no. 1, pp. 90-102, 1996.

[2] H. Schulte Jr, "The characteristics of the mckibben artificial muscle. application of external power in prosthetics and orthotics, washington dc," Nat. Acad. Sci.-Nat. Res. Council, 1961.

[3] F. Ilievski, A. D. Mazzeo, R. F. Shepherd, X. Chen, and G. M. Whitesides, "Soft robotics for chemists," Angewandte Chemie, vol. 123, no. 8, pp. 1930-1935, 2011.

[4] J. Bishop-Moser, G. Krishnan, C. Kim, and S. Kota, "Design of soft robotic actuators using fluid-filled fiber-reinforced elastomeric enclosures in parallel combinations," in Intelligent Robots and Systems (IROS), 2012 IEEE/RSJ International Conference on, pp. 4264-4269, IEEE, 2012.

[5] J. Bishop-Moser, G. Krishnan, and S. Kota, "Force and moment generation of fiber-reinforced pneumatic soft actuators," in Intelligent Robots and Systems (IROS), 2013 IEEE/RSJ International Conference on, pp. 4460-4465, IEEE, 2013.

[6] M. Luo, W. Tao, F. Chen, T. K. Khuu, S. Ozel, and C. D. Onal, "Design improvements and dynamic characterization on fluidic elastomer actuators for a soft robotic snake," in Proceedings of the IEEE International Conference on Technologies for Practical Robot Applications, 2014.

[7] M. Luo, M. Agheli, and C. D. Onal, "Theoretical modeling and experimental analysis of a pressure-operated soft robotic snake," Soft Robotics, vol. 1, no. 2, pp. 136-146, 2014.

[8] R. F. Shepherd, F. Ilievski, W. Choi, S. A. Morin, A. A. Stokes, A. D. Mazzeo, X. Chen, M. Wang, and G. M. Whitesides, "Multigait soft robot," Proceedings of the National Academy of Sciences, vol. 108, no. 51, pp. 20400-20403, 2011.

[9] C. D. Onal and D.Rus, "Autonomous undulatory serpentine locomotion utilizing body dynamics of a fluidic soft robot," Bioinspiration \& biomimetics, vol. 8, no. 2, p. 026003, 2013.

[10] A. D. Marchese, K. Komorowski, C. D. Onal, and D. Rus, "Design and control of a soft and continuously deformable $2 \mathrm{~d}$ robotic manipulation system," in Proceedings of IEEE International Conference on Robotics and Automation, 2014.

[11] A. Gentile, N. Giannoccaro, and G. Reina, "Experimental tests on position control of a pneumatic actuator using on/off solenoid valves," in Industrial Technology, 2002. IEEE ICIT'02. 2002 IEEE International Conference on, vol. 1, pp. 555-559, IEEE, 2002.

[12] R. B. Van Varseveld and G. M. Bone, "Accurate position control of a pneumatic actuator using on/off solenoid valves," Mechatronics, IEEE/ASME Transactions on, vol. 2, no. 3, pp. 195-204, 1997.

[13] M.-C. Shih and M.-A. Ma, "Position control of a pneumatic cylinder using fuzzy pwm control method," Mechatronics, vol. 8, no. 3, pp. 241-253, 1998.

[14] K. Ahn and S. Yokota, "Intelligent switching control of pneumatic actuator using on/off solenoid valves," Mechatronics, vol. 15, no. 6, pp. 683-702, 2005.

[15] A. Messina, N. I. Giannoccaro, and A. Gentile, "Experimenting and modelling the dynamics of pneumatic actuators controlled by the pulse width modulation (pwm) technique," Mechatronics, vol. 15, no. 7, pp. 859-881, 2005.

[16] A. K. Paul, J. Mishra, and M. Radke, "Reduced order sliding mode control for pneumatic actuator," Control Systems Technology, IEEE Transactions on, vol. 2, no. 3, pp. 271-276, 1994.

[17] E. J. Barth, J. Zhang, and M. Goldfarb, "Sliding mode approach to pwm-controlled pneumatic systems," in American Control Conference, 2002. Proceedings of the 2002, vol. 3, pp. 2362-2367, IEEE, 2002.

[18] Ç. D. Önal, K. S. Abidi, and A. Şabanoviç, "A cascaded sliding mode hybrid force/position controller," 2005.

[19] V. Utkin, "Variable structure systems with sliding modes," IEEE Transactions on Automatic Control, vol. 22, no. 2, pp. 212-222, 1977. 